\title{
PREVALENCIA DE MALESTAR PSICOLÓGICO EN ESTUDIANTES DE ENFERMERÍA RELACIONADA CON FACTORES SOCIODEMOGRÁFICOS, ACADÉMICOS Y FAMILIARES
}

\section{PREVALENCE OF PSYCHOLOGICAL DISTRESS IN NURSING STUDENTS ASSOCIATED WITH SOCIODEMOGRAPHIC, ACADEMIC AND FAMILY FACTORS}

\author{
Luz María Herrera L. * \\ María Soledad Rivera M. ${ }^{* *}$
}

\begin{abstract}
RESUMEN
Estudio descriptivo correlacional realizado con el objetivo de medir la prevalencia y nivel de síntomas de malestar psicológico y su relación con variables sociodemográficas, familiares y académicas, en estudiantes de pregrado de la carrera de Enfermería. Se aplicó el Cuestionario de Salud General de Goldberg de 12 ítemes a 228 estudiantes, previo consentimiento informado. Entre los resultados, se obtuvo una prevalencia de $36 \%$ de malestar psicológico; los síntomas más frecuentes fueron: agobio y tensión, falta de concentración, menor capacidad para disfrutar de la vida diaria, sentirse deprimido, pérdida de sueño e incapacidad para enfrentar problemas. El malestar psicológico se asoció significativamente con: menor tiempo destinado a recreación, preocupación económica, más de diez horas diarias de estudio personal, procedencia de provincia, percepción de bajo nivel socioeconómico, percepción de pobre apoyo familiar, promedio de notas inferior a cinco y mediana satisfacción con la carrera. Se concluye que existe una alta prevalencia de malestar psicológico entre los estudiantes de enfermería, relacionado con características propias del estudiante de orden personal, académico y familiar.
\end{abstract}

Palabras clave: Salud mental, trastornos de ansiedad, depresión, trastornos del humor, estudiantes de enfermería.

\begin{abstract}
Descriptive correlational study conducted with the aim to measure the prevalence and level of psychological distress symptoms and its relationship with sociodemographic, family and academic variables, in undergraduate Nursing students. The Goldberg General Health Questionnaire of 12 items was applied to 228 students, previous informed consent, finding a 36\% prevalence of psychological distress. The most frequent symptoms were: overwhelmed and tension, lack of concentration, reduced ability to enjoy daily life, feeling depressed, loss of sleep and incapacity to cope with problems. The psychological discomfort was significantly associated with: less time for recreation, economic concern, more than ten hours of personal study, mostly coming from province, low socioeconomic level perception, poor family support perception, average grade below five and medium satisfaction with the career. It was concluded that there is a high prevalence of psychological distress among students of nursing related to student's personal, academic and family characteristics.
\end{abstract}

Key words: Mental health, anxiety disorders, depression, mood disorders, students, nursing.

Fecha recepción: 13.09.10 Fecha aceptación: 26.05.11

\footnotetext{
* Enfermera Matrona, Magíster en Enfermería. Profesora Asistente Departamento Salud del Niño y Adolescente. Escuela de Enfermería, Facultad de Medicina, Pontificia Universidad Católica de Chile. Santiago, Chile. Email: luzma@uc.cl.

${ }^{* *}$ Doctora en Enfermería. Profesor Asociado, Departamento Salud de la Mujer, Escuela de Enfermería, Facultad de Medicina, Pontificia Universidad Católica de Chile. Email: mriverma@uc.cl.
} 


\section{INTRODUCCIÓN}

La formación de enfermería se caracteriza por una exigente preparación en el área de las ciencias de la salud y en el ámbito profesional, lo que se acompaña de altos niveles de estrés (1-3).

Durante su proceso de formación (5 años) se espera que los estudiantes de enfermería desarrollen competencias de alta complejidad para lograr desempeñar su rol profesional, de acuerdo a lo establecido en el artículo 113 del Código Sanitario de Chile (4). Los desafíos que plantea esta responsabilidad multidimensional, demanda profesionales de enfermería con competencias cada vez más integrales y complejas, especialmente en la dimensión psicosocial, como son: la relación de ayuda con el paciente y su familia en situaciones críticas, acompañamiento en el sufrimiento y en la fase terminal de la vida, trabajo en equipo y liderazgo de equipos de enfermería o multidisciplinarios, entre otras.

La exposición mantenida a factores estresantes en los estudiantes puede producir deterioro del funcionamiento normal del organismo generando, entre otros, falta de concentración, dificultad para memorizar, para resolver problemas, déficits en las habilidades de estudio, escasa productividad y un menor rendimiento académico (5). Por otro lado, diversos estudios epidemiológicos y sociales han demostrado consistentemente que el estrés psicosocial asociado a condiciones adversas de vida se relaciona con un aumento de trastornos mentales, particularmente ansiedad y depresión, y además constituye un factor de riesgo para los trastornos por abuso y dependencia a sustancias adictivas $(6,7)$.

El malestar psicológico, entendido como el nivel de estrés, desmoralización, disconfort y desasosiego percibido en sí mismo (8), se ha relacionado con una serie de factores de orden sociodemográfico, académico y familiar en los estudiantes del área de la salud. Entre los factores sociodemográficos se describen la menor edad y nivel socioeconómico bajo (3, 9-11). Los factores académicos incluyen las evaluaciones, exámenes y sobrecarga de estudio $(12,13)$, la insatisfacción con la carrera (14), cursar el primer y tercer año de carrera $(9,11)$; y entre los factores familiares se reportan el vivir sin los padres (15) y una pobre relación familiar $(16,17)$.

Se supone que los estudiantes con "buena salud mental" responden al medio proactivamente adaptándose a las condiciones adversas, enfrentan y resuelven exitosamente sus conflictos personales e interpersonales y mantienen relaciones interpersonales cordiales en su ambiente de trabajo $(18,19)$. Es decir, cabe esperar por un lado que los estudiantes de enfermería sean capaces de responder "saludablemente" a los desafíos que les presenta la naturaleza propios del cuidado de enfermería, en la cual la enfermera se utiliza a sí misma como herramienta de ayuda para sus pacientes que viven complejas experiencias de dolor y sufrimiento. Por otro lado, se espera que además se adapten a las condiciones desfavorables de trabajo, tan comunes en los servicios de salud actuales en los países en vías de desarrollo (horarios de trabajo que interfieren en su desarrollo personal y familiar, conflicto y ambigüedad de las funciones, sueldos inadecuados, presión asistencial y emocional, entre otros) (20).

En Chile, se ha abordado escasamente el problema de salud mental de los estudiantes de enfermería y se ha hecho a través de la medición de los niveles de estrés, reportándose prevalencias de $40 \%$ en Universidad de los Andes (21), y 65,7\% en la Universidad Austral de Chile en Valdivia ${ }^{1}$. No se han encontrado estudios que midan el malestar psicológico en estudiantes de enfermería en

\footnotetext{
${ }^{1}$ Burgos MF. Calidad de vida y estrés de los estudiantes de la Escuela de Enfermería. Tesis de grado, Universidad Austral de Chile, 2003.
} 
Chile, pero sí en estudiantes de medicina de la Pontificia Universidad Católica de Chile (11), el que reporta una prevalencia de $40,68 \%$ con la aplicación del Cuestionario de Salud General de Goldberg, en su versión de 12 ítemes.

La motivación de las autoras de esta investigación surge de la experiencia de una de ellas, en la atención de estudiantes con problemas de rendimiento y fracaso académico, asociados a un nivel importante de sufrimiento psicológico.

Debido a que ha sido escasa la atención puesta en este fenómeno en Chile, la presente investigación tiene como propósito contribuir en la construcción de conocimiento sobre este problema. El objetivo general de este estudio es determinar prevalencia de malestar psicológico y su relación con características socioeconómicas, familiares y académicas de los estudiantes de enfermería de la Pontificia Universidad Católica de Chile, durante el segundo semestre de 2006.

\section{MATERIAL Y MÉTODO}

Este estudio cuantitativo-descriptivo correlacional fue realizado en estudiantes regulares de la carrera de enfermería de la Pontificia Universidad Católica de Chile (UC), durante el segundo semestre de 2006. El universo corresponde a 508 estudiantes, incluidos aquéllos con suspensión de estudios por problemas de salud. La muestra final de 228 estudiantes que aceptaron participar en el estudio corresponde al 45\% del universo y se obtuvo de forma aleatoria. La distribución porcentual por año académico fue de 19,7\% de primer año, $17,1 \%$ de segundo, $24,1 \%$ de tercero; $21,1 \%$ de cuarto y $18 \%$ de quinto.

Se contó con la aprobación tanto de la Dirección como de la Comisión de Ética de la Dirección de Investigación de la Escuela de Enfermería UC. Los estudiantes que obtuvieran un nivel de malestar psicológico su- gerente de trastorno de salud mental ( $\geq$ a 5 síntomas) tuvieron la opción de consultar para evaluación y eventual tratamiento en la Unidad de Apoyo Psicológico de la Universidad. Los resultados estuvieron disponibles para los estudiantes en forma semanal y fueron entregados en un sobre cerrado bajo el seudónimo del estudiante. Se garantizó a los participantes el resguardo de sus derechos mediante la observación de los principios éticos universales para toda investigación con seres humanos (22). El proceso de recolección de datos se realizó, en un periodo 11 semanas, mediante el envío del instrumento vía online.

Para medir el nivel de malestar psicológico se aplicó el instrumento Cuestionario de Salud General de Goldberg (1978), versión de 12 ítemes, diseñado con el fin de detectar trastornos mentales (no psicóticos) en el contexto comunitario, validado en Chile (23). Los coeficientes de confiabilidad alfa de Cronbach oscilan entre 0,82 y 0,95 (24) y en el presente estudio fue de 0,90 .

El cuestionario consta de doce proposiciones con 4 alternativas de respuesta en escala de Lickert (mejor que lo habitual, igual que lo habitual, menos que lo habitual y mucho menos que lo habitual). Para la calificación de las puntuaciones del test se usó el método de puntuación original de Goldberg (GHQ), que consiste en asignar cero punto a las dos primeras respuestas y un punto a las dos últimas (24). De esta manera el cuestionario arroja un puntaje ordinal que va de cero a doce puntos. El puntaje umbral para determinar los "probables casos" de malestar psicológico en la presente investigación fue $\geq \mathrm{a}$ 5 puntos, al igual que el estudio de referencia (11).

Para medir las variables sociodemográficas, familiares y académicas se diseñó un cuestionario con preguntas de respuesta cerrada. Las variables sociodemográficas estudiadas fueron: edad, género, estado civil, número de hijos, percepción de nivel socioeconómico (alto, medio, bajo), preocupación 
económica, trabajo part-time, procedencia de provincia, y tiempo destinado a recreación; las variables académicas: semestre académico, carga académica (número de créditos inscritos en el semestre actual), promedio ponderado acumulado de notas, horas de estudio personal y satisfacción con la carrera; y las variables familiares: vivir con o sin los padres y apoyo familiar percibido (sentirse querido, apoyo frente a problemas y apoyo frente a la toma de sus decisiones).

Para el análisis de los datos se utilizó el software estadístico SSPS 10.0 y la planilla de cálculo Excel. Se realizó análisis descriptivo de frecuencias, mediana, rango y desviación estándar. Para el análisis correlacional, debido a que la variable malestar psicológico no se distribuyó normalmente (asimetría de 0,711), se utilizaron los estadígrafos Chi cuadrado y coeficiente de Spearman. El nivel de significación estadística considerado para este estudio fue $\alpha=0,05$.

\section{RESULTADOS}

La muestra se caracteriza por un predominio de mujeres $(96,1 \%)$, solteras(os) $(94,3 \%)$ y sin hijos (91,2\%), con un promedio de edad de 21,9 años. La mayoría de los participantes $(61,4 \%)$ percibe que pertenece al nivel socioeconómico medio, el 22,4\% al bajo y el $16,2 \%$ al alto. El 54,8\% manifiesta preocupación por su situación económica. El 19,2\% proviene de provincia y el $22 \%$ trabaja parttime un promedio de 18,2 (DS=14,5) h semanales. El tiempo promedio que destinan a actividades recreativas es de 8,05 (DS=7,6) h semanales.

Los participantes tienen un Promedio Ponderado Acumulado de notas (PPA) de $5,3$ (DS $=0,41)$; una carga académica semestral promedio de 49 créditos y dedican un promedio de $4,01(\mathrm{DS}=3,51) \mathrm{h}$ diarias al estudio personal. El 58,3\% reporta estar totalmente satisfecho con la carrera de enfermería, el resto está entre medianamente satisfecho $(37,3 \%)$ y totalmente insatisfecho $(3,9 \%)$. La mayoría de los participantes $(73,2 \%)$ vive con uno o ambos padres; el resto $(26,8 \%)$ no vive con ellos, residiendo ya sea con otros familiares, amigos, pareja, hermanos, solo, hogar universitario o pensión. Un porcentaje no despreciable percibe que "a veces/nunca" se siente querido, apoyado frente a sus problemas, y apoyado en la toma de decisiones $(12,7 \%, 20,1 \%$ y $24,6 \%$, respectivamente).

El 36\% de los participantes presenta malestar psicológico con un promedio de síntomas de 7,8 (DS=2,2) (Tabla 1). Se observa un aumento sostenido en la prevalencia de malestar psicológico desde primer a cuarto

Tabla 1. Resultados globales test de Golberg, según año académico.

\begin{tabular}{|l|cc|cc|cc|}
\hline \multicolumn{1}{|c|}{ Año Académico } & \multicolumn{2}{|c|}{$\begin{array}{c}\text { Muestra } \\
\text { Puntaje GHQ }\end{array}$} & \multicolumn{2}{c|}{$\begin{array}{c}\text { "Posibles casos" } \\
\text { Puntaje GHQ }<5\end{array}$} & \multicolumn{2}{c|}{$\begin{array}{c}\text { "Posibles casos" } \\
\text { Puntaje GHQ } \geq 5\end{array}$} \\
\hline & $\mathrm{n}$ & $\mathrm{M}(\mathrm{DS})$ & $\mathrm{n}$ & $\mathrm{M}(\mathrm{DS})$ & $\mathrm{n}$ & $\mathrm{M}(\mathrm{DS})$ \\
Primero & 45 & $3,7(3,2)$ & 32 & $2,0(1,6)$ & 13 & $7,9(2,1)$ \\
Segundo & 39 & $3,6(3,2)$ & 27 & $1,8(1,5)$ & 12 & $7,6(2,3)$ \\
Tercero & 55 & $4,1(3,2)$ & 34 & $2,0(1,5)$ & 21 & $7,5(2,2)$ \\
Cuarto & 48 & $4,4(3,9)$ & 27 & $1,5(1,4)$ & 21 & $8,2(2,4)$ \\
Quinto & 41 & $4,2(3,2)$ & 26 & $2,2(1,6)$ & 15 & $7,7(2,2)$ \\
Total & 228 & $4,0(3,4)$ & 146 & $1,9(1,5)$ & 82 & $7,8(2,2)$ \\
\hline
\end{tabular}


año de carrera, con un moderado descenso en quinto $(28,9 \%, 30,8 \%, 38,2 \%, 43,7 \%$ y $36,6 \%$, respectivamente). La diferencia de frecuencias observada entre los cursos no es estadísticamente significativa. Los resultados por ítemes del test arrojan que el 68\% se percibe con agobio y tensión constante, el $50 \%$ con problemas de concentración, el $42,7 \%$ con menor capacidad para disfrutar de la vida diaria, el 41,7\% refiere sentirse deprimido, el $40 \%$ pérdida de sueño y el $37,3 \%$ menor capacidad para enfrentar problemas. La frecuencia del resto de los ítemes se observa en el Gráfico 1.

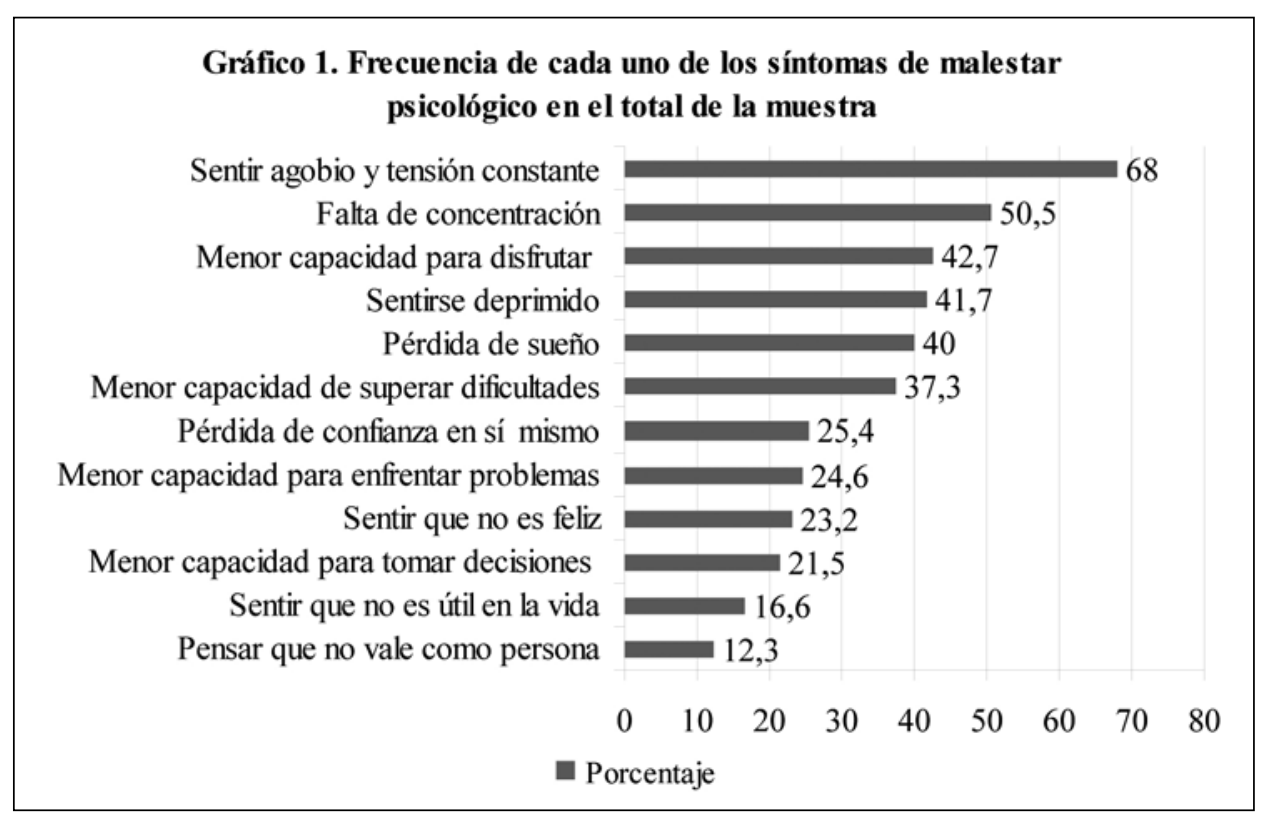

El análisis correlacional permite establecer asociación estadísticamente significativa entre el malestar psicológico y las siguientes variables sociodemográficas, académicas y familiares:

- Tiempo de recreación: a menor tiempo de recreación mayor malestar psicológico ( $r s=-0,26, p=0,0001)$.

- Preocupación económica: se observa mayor malestar psicológico entre los estudiantes que manifiestan preocupación por su situación económica en comparación con aquellos a quienes no les preocupa $\left(\chi^{2}(1,228)=6,29, p=0,001\right)$.

- Percepción de nivel socioeconómico: los estudiantes de nivel socioeconómico bajo presentan proporcionalmente mayor fre- cuencia de malestar psicológico que aquéllos de nivel socioeconómico medio y alto $\left(\chi^{2}(1,51)=6,38, p=0,01\right)$.

- Procedencia de provincia: aquellos estudiantes que provienen de provincia presentan mayor malestar psicológico en comparación con aquellos que son de Santiago $\left(\chi^{2}(1,228)=4,55, p=0,03\right)$.

- Horas de estudio personal: existe mayor frecuencia de malestar psicológico en aquellos que dedican más de 10 horas diarias al estudio personal $\left(\chi^{2}(1,17)=6,24\right.$, $p=0,01)$.

- Promedio ponderado acumulado de notas: se observa una diferencia estadísticamente significativa entre los estudiantes con promedio de notas menor 5 en comparación con aquéllos con promedio de 
notas $>$ a $5\left(\chi^{2}(1,225)=4,55, p=0,03\right)$.

- Satisfacción con la carrera: los estudiantes que manifiestan mediana satisfacción con la carrera presentan mayor frecuencia de malestar psicológico que aquéllos totalmente satisfechos $\left(\chi^{2}(1,218)=4,113, p\right.$ $=0,04)$.

- Apoyo familiar percibido: la condición de sentirse "a veces/nunca" queridos por su familia se asocia con mayor malestar psicológico $\left(\chi^{2}(1,29)=4,65, p=0,03\right)$. Lo mismo sucede con aquellos que manifiestan apoyo "a veces/nunca" frente a sus problemas $\left(\chi^{2}(1,46)=5,25, p=0,02\right)$.

Los estudiantes que viven con sus padres presentan menor malestar psicológico en comparación con aquellos que viven sin ellos (31,9\% vs 46\%). Sin embargo, esta diferencia de frecuencia no es estadísticamente significativa.

Por último, no se observa asociación estadísticamente significativa entre malestar psicológico y las variables edad, trabajo parttime, año académico que cursa y carga académica. Dada la baja frecuencia de hombres, de estudiantes casados y de estudiantes sin hijos en la muestra no se realizó análisis correlacional según sexo, estado civil y número de hijos.

\section{DISCUSIÓN Y COMENTARIO}

Se obtuvo una alta prevalencia de malestar psicológico en las estudiantes de enfermería (36\%) con un nivel de síntomas sugerente de un probable problema de salud mental. Dicha prevalencia es semejante a la encontrada en estudiantes de medicina en Chile y de enfermería en Australia $(11,25)$. Entre los síntomas más frecuentes se destacan "sentirse deprimido" $(41,7 \%)$ y "menor capacidad para disfrutar de la vida diaria" $(42,7 \%)$, síntomas que califican para sospecha de una probable depresión (26). Varios estudios realizados en América Latina, Europa y Norteamérica también informan de altas prevalencias de riesgo de depresión, entre 22 y $41 \%$ en estudiantes de enfermería (12, 27-30).

La mayor prevalencia de malestar psicológico se encuentra en los estudiantes de tercer, cuarto y quinto año de carrera $(38,2 \%$, $43,7 \%$ y $36,6 \%$, respectivamente). Considerando la literatura $(1,3,25,31)$, es posible explicar que esta condición esté relacionada con las situaciones altamente estresantes que enfrentan los estudiantes de enfermería durante sus experiencias clínicas, tales como: contacto con el dolor, sufrimiento y muerte; vivir situaciones urgentes y graves para las que muchas veces no se sienten preparados; miedo a cometer errores, entre otras (31). El descenso observado en quinto año podría tener relación con un proceso de adaptación y empoderamiento en el rol profesional, experimentado por el estudiante en su último año de carrera (9).

En este estudio, el malestar psicológico se asocia significativamente con: menor tiempo destinado a recreación, mayor tiempo de estudio personal, promedio de notas $<5$, procedencia de provincia, nivel socioeconómico bajo, preocupación económica, mediana satisfacción con la carrera y pobre relación familiar. El hecho que los estudiantes que presentan malestar psicológico destinen menos tiempo a la recreación y más tiempo al estudio personal, se podría explicar porque la alteración del ánimo se acompaña generalmente de pérdida de interés por un lado y de menor capacidad de concentración por otro, requiriendo mayor tiempo de estudio para lograr el rendimiento académico esperado por ellos. La asociación significativa con un bajo promedio de notas también ha sido observada en otros estudios realizados en estudiantes universitarios $(5,32)$. La mayor frecuencia de malestar psicológico entre los estudiantes que proceden de provincia podría explicarse por tener reducida su red de apoyo familiar y social. 
La asociación con preocupación económica y bajo nivel socioeconómico autopercibido también ha sido reportada en estudios realizados en estudiantes de enfermería de Colombia y Reino Unido (14, 32). Lo mismo sucede con el factor insatisfacción con la carrera observado en la población universitaria de una universidad de Colombia (14); y con una pobre relación familiar, en universitarios de Asia y Colombia $(16,17)$.

A diferencia de otros estudios, las variables que no correlacionaron significativamente con malestar psicológico son edad, trabajo part-time, carga académica y vivir sin los padres. Por ejemplo, en relación a la edad, Benítez (11) en Chile y Souza y Menezes (9) en Brasil reportan correlación inversa con la presencia de problemas de salud mental. Carney et al. (33) observaron que en los estudiantes universitarios de la Universidad de Glasgow (incluidos enfermería y medicina) el trabajo part-time tiene un efecto perjudicial significativo en la salud mental y Facundes y Ludermir (15), que tanto la sobrecarga académica (más de 30 horas de clases a la semana) como vivir sin los padres se asocia con los trastornos del ánimo en estudiantes del área de la salud colombianos.

Por último, en relación a las limitaciones de este estudio, se plantea que el test de screening empleado en este estudio puede no identificar correctamente estudiantes con problemas crónicos de salud mental, ya que se pregunta por la presencia de síntomas actuales comparados con "lo habitual". Entonces, si la respuesta es lo habitual frente a un síntoma, ésta no se cuenta como indicadora de síntoma. Ello podría haber influido en la prevalencia de "probables casos" (34).

Las principales conclusiones del estudio son:

- Existe alta prevalencia de malestar psicológico $(36 \%)$ con un nivel de síntomas sugerente de un probable problema de salud mental en los estudiantes de enfermería de la UC.
- La mayor prevalencia de malestar psicológico se encuentra en los estudiantes de tercer, cuarto y quinto año de carrera. Considerando la literatura, es probable hipotetizar que esta condición esté relacionada con las situaciones (altamente estresantes) que enfrentan durante sus experiencias clínicas y a deficiencias de estrategias de afrontamiento. Sin embargo, este estudio no permite confirmar esta relación, por lo que se sugiere realizar una nueva investigación con este fin.

- El malestar psicológico se asocia significativamente con: menor tiempo destinado a recreación, mayor tiempo de estudio personal, procedencia de provincia, nivel socioeconómico bajo, preocupación económica, mediana satisfacción con la carrera y pobre relación familiar.

- De todos estos factores relacionados con el malestar psicológico, se destaca la satisfacción con la carrera. Ello, porque tiene implicancias prácticas relacionadas con la formación de la identidad profesional y compromiso con el ejercicio profesional futuro. Este hallazgo se considera relevante y amerita una nueva investigación, con el fin de conocer en profundidad tanto las motivaciones y expectativas de los estudiantes como los factores que están influyendo en ello. Con dichos resultados se podría iniciar un proceso de reflexión académica, en conjunto con los estudiantes, que culmine con la implementación de estrategias que permitan aumentar la satisfacción con la carrera.

- No se encontró relación estadísticamente significativa con: edad, trabajo part-time, tiempo de traslado a la universidad, carga académica y vivir con o sin los padres.

El alto nivel de malestar psicológico encontrado entre los estudiantes de enfermería (36\%) deja en evidencia una realidad que debe ser considerada por las autoridades de la escuela en los planes destinados a velar por el bienestar de los alumnos. Las autoras de 
este estudio creen en la misión de las escuelas de enfermería de formar jóvenes enfermeras/ os que hagan una diferencia en su acción social profesional transformadora, en beneficio de las personas que cuidan y de la sociedad, sin menoscabo de su propia salud mental. En este sentido se sugiere desarrollar un proceso de reflexión académica que se concrete en la toma de decisiones relacionadas con la implementación de estrategias viables y sistemáticas, que favorezcan la mantención del bienestar psicológico en los estudiantes.

\section{REFERENCIAS}

1. Zupiria X, Huitzi X, Alberdi MJ, Uranga MJ, Eizmendi I, Barandiaaran M, et al. Stress sources in nursing practice. Evolution during nursing training. Nurse Educ Today. 2007; 27(7): 777-787.

2. Tully A. Stress, sources of stress and ways of coping among psychiatric nursing students. J Psychiatric Ment Health Nursing. 2004; 11(1): 43-47.

3. Timmins F, Kaliszer M. Aspects of nurse education programmes that frequently cause stress to nursing students: fact-finding sample survey. Nurse Educ Today. 2002; 22(3): 203-211.

4. Código Sanitario República de Chile. Santiago, Chile: Jurídica de Chile. 1998.

5. Pérez M, Rodríguez M, Borda M, Del Río C. Estrés y rendimiento académico en estudiantes universitarios. Cuadernos de Medicina Psicosomática y Psiquiatría de Enlace. 2003; 67-68: 26-33.

6. Trucco M. Estrés y trastornos mentales: aspectos neurobiológicos y psicosociales. Rev Chil Neuro-Psiquiat. 2002; 40 (supl.2): 8-19.

7. Caldwell TM, Rodgers B, Jorm AF, Christensen $\mathrm{H}$, Jacomb PA, Korten AE, et al. Patterns of association between alcohol consumption and symptoms of depression and anxiety in young adults. Addic- tion. 2002; 97(5): 583-595.

8. Espíndola JG, Morales F, Díaz E, Pimentel , Meza P, Henales C, et al. Malestar psicológico: algunas de sus manifestaciones clínicas en la paciente gineco-obstétrica hospitalizada. Perinatol Reprod Hum. 2006; 20(4): 112-122.

9. Souza F, Menezes M. Estresse nos estudantes de medicina da Universidad Federal do Ceará, Brasil. Rev bras educ méd. 2005; 29(2): 91-96.

10. Sender R, Salamero M, Valles A, Valdés M. Psychological variables for identifying suseptibility to mental disorders in medical students at the University of Barcelona. Medical Education Online [Internet]. 2004 [citado 20 Oct 2007]; 9(9): 1-5. Disponible en http://www.med-ed-online. org/res00088.htm

11. Benítez C, Quintero J, Torres R. Prevalencia de riesgo de trastornos psiquiátricos en estudiantes de pregrado de la Escuela de Medicina de la P. Universidad Católica de Chile. Rev Med Chile. 2001;129(2): 173-178.

12. Alves-Apóstolo J, Alves M, Pineda J. Evaluación de los estados emocionales de estudiantes de enfermería. Índex de Enfermería [Internet]. 2007 [citado 20 Oct 2007]; 16(56): 26-29. Disponible en http://dialnet.unirioja.es/

13. Evans W, Kelly B. Pre-registration diploma student nurse stress and coping measures. Nurse Educ Today. 2004; 24(6): 473-482.

14. Amézquita ME, González R, Zuluaga D. Prevalencia de la depresión, ansiedad y comportamiento suicida en la población estudiantil de pregrado de la Universidad de Caldas, año 2000. Rev Colomb Psiquiatr. 2003; 32(4):341-356.

15. Facundes VLD, Ludermir AB. Common mental disorders among health care students Rev Bras Psiquiat. 2005; 27(3): 194-200.

16. Mohd Sidik S, Rampal L, Kaneson N. Prevalence of emotional disorders 
among medical students in a Malaysian university. Asia Pac Fam Med. 2003; 2(4): 213-217.

17. Gaviria S, Rodríguez M, Álvarez T. Calidad de la relación familiar y depresión en estudiantes de medicina de Medellín, Colombia, 2000. Rev Chil Neuro-Psiquiat. 2000; 40(1): 41-46.

18. Organización Mundial de la Salud. Informe sobre la salud en el mundo 2001. Salud mental: nuevos conocimientos, nuevas esperanzas. Ginebra, Suiza: $\mathrm{Pu}-$ blicaciones OMS; 2001.

19. Sánchez E, Álvaro JL. Medición de la salud mental. Rev Chil Salud Pública. 2002; 6(1): 41-51.

20. Consejo Internacional de Enfermeras. El CIE aborda el estrés en el trabajo y la amenaza que supone para la salud del trabajador [monografía en Internet]. Ginebra, Suiza: Consejo Internacional de Enfermeras; 2001[citado 5 Oct 2006]. Disponible en: http://www.icn. ch/images/stories/documents/publications/fact_sheets/19h_FS-Estres_trabajo_amenaza_salud_trabajador-Sp.pdf

21. Marty M, Lavín M, Figueroa M, Larraín D, Cruz C. Prevalencia de estrés en estudiantes del área de la salud de la Universidad de los Andes y su relación con enfermedades infecciosas. Rev. Chil. Neuro-Psiquiat. 2005; 43(1):25-32.

22. Office of Human Subjects Research of the National Institutes of Health [Internet]. The Belmont Report. Ethical Principles and Guidelines for the protection of human subjects of research. Bethesda: National Institutes of Health; 1979 [citado 10 enero 2007]. Disponible en: http:// ohsr.od.nih.gov/guidelines/

23. Araya R, Wynn R, Lewis G. Comparison of two self-administered psychiatric questionnaires (GHQ-12 and SRQ-20) in primary care in Chile. Soc Psychiatry Psychiatr Epidemiol. 1992; 27(4):168-73.

24. Lobo A, Muñoz P. Cuestionario de salud general. Guía para el usuario de las distintas versiones. Versiones en lengua española validadas. Barcelona: Masson; 1996.

25. Lo R. A longitudinal study of perceived level of stress, coping and self-steem of undergraduate nursing students: an Australian case study. Journal of Advance Nursing. 2002; 39(2): 119-126.

26. Ministerio de Salud Chile [Internet]. Guía clínica GES para la depresión en personas de 15 años y más. Santiago, Chile: MINSAL; 2009 [citado 7 abril 2010]. Disponible en: http://www.minsal.gob. cl/portal/url/item/7222754637c08646e0 4001011f014e64.pdf

27. Olmedo-Buenrostro B, Torres-Hernández J, Velasco-Rodríguez R, Mora-Brambila A, Blas-Vargas L. Prevalencia y severidad de depresión en estudiantes de enfermería de la Universidad de Colima. Rev Enferm IMSS. 2006; 14(1): 17-22.

28. Garro IMB, Camillo SO, Nobrega MPSS. Depressao em graduandos de enfermagem. Acta Paulista de Enferm. 2006; 19(2):62-167.

29. Santos TM, Almeida AO, Martins HO, Moreno V. Aplicação de um instrumento de avaliação do grau de depressão em universitários do interior paulista durante a graduação em Enfermagem. Acta Sci Health Sci. 2003; 25(2):171-6.

30. Kim K. Baccalaureate nursing students' experiences of anxiety producing situations in the clinical setting. Contemp Nurse. 2003; 14(2):145-55.

31. Letelier P, Valenzuela S. Violencia: fenómeno relevante de estudio en campos clínicos intra-hospitalarios de enfermería. Cien enferm. 2002; 8(2): 21-26.

32. Andrews B, Wilding J. The relation of depression and anxiety to life-stress and achievement in students. Br J Psychol. 2004; 95(4): 509-521.

33. Carney C, McNeish S, McColl J. The impact of part time employment on students health and academic performance: a Scottish perspective. Journal 
of Futher \& Higher Education. [Internet]. 2005 [citado 20 Oct 2007]; 29(4): 307-319. Disponible en http://pdfserve.informaworld.com.ezproxy.puc. cl/162051_751315603_727324933.pdf
34. Trucco M, Campusano ME, Larraín S. Un cuestionario para detectar desórdenes emocionales. Estudio de validación preliminar. Rev Chil Neuro-Psiquiat.1979; 17(1): 20-25. 